\title{
Nonlinear QCD evolution: Saturation without unitarization
}

\author{
Alexander Kovner \\ Department of Mathematics and Statistics, University of Plymouth, 2 Kirkby Place, Plymouth, PL4 8AA, United Kingdom
}

Urs Achim Wiedemann

Theory Division, CERN, CH-1211 Geneva 23, Switzerland

(Received 17 December 2001; published 30 September 2002)

\begin{abstract}
We consider the perturbative description of saturation based on the nonlinear QCD evolution equation of Balitsky and Kovchegov. Although the nonlinear corrections lead to saturation of the scattering amplitude locally in impact parameter space, we show that they do not unitarize the total cross section. The total cross section for the scattering of a strongly interacting probe on a hadronic target is found to grow exponentially with rapidity $t=\ln \left(s / s_{0}\right), \sigma \propto \exp \left\{\left(\alpha_{s} N_{c} / 2 \pi\right) \epsilon t\right\}$ where $\epsilon$ is a number of order unity. The origin of this violation of unitarity is the presence of long range Coulomb fields away from the saturation region. The growth of these fields with rapidity is not tempered by the nonlinearity of the Balitsky-Kovchegov equation.
\end{abstract}

DOI: 10.1103/PhysRevD.66.051502

Understanding the growth of total scattering cross sections with energy $\sqrt{s}$ is a long standing problem. The unitarity, or Froissart, bound states that the total inelastic cross section for the scattering of a hadronic projectile on a hadronic target cannot grow faster than $\sigma<\pi d^{2} t^{2}$, where $d$ is some typical hadronic scale and $t=\ln \left(s / s_{0}\right)$ is the rapidity. While QCD is a unitary theory and therefore satisfies this unitarity bound, there is no guarantee that perturbative calculations preserve this property. In fact, the linear perturbative Balitsky-Fadin-Kuraev-Lipatov (BFKL) evolution equation implies an exponential growth of $\sigma$ with $t$, thus violating unitarity.

Following the pioneering works of [1], there has been recent progress in high energy hadronic scattering in a derivation of a nonlinear evolution equation [2-4] which tames the BFKL-type growth. These equations resum the nonlinear corrections to the QCD evolution with rapidity (energy) to all orders in partonic density and to first order in the QCD coupling. While previous studies of these equations apply to systems with translational invariance in the impact parameter plane, here we explore for the first time their impact parameter dependence. For the equations first derived by Balitsky [2], we show that the total cross section does not unitarize but grows exponentially with $t$ [5].

We will use the form of the evolution equations first given by Weigert [4]:

$$
\begin{aligned}
\frac{d U(x)}{d t}= & g U(x) i T^{a} \int \frac{d^{2} z}{\sqrt{4 \pi^{3}}} \frac{(x-z)_{i}}{(x-z)^{2}} \\
& \times\left[1-\widetilde{U}^{\dagger}(x) \widetilde{U}(z)\right]^{a b} \xi_{i}^{b}(z) \\
& +\frac{\alpha_{s}}{2 \pi^{2}} U(x) T^{a} \int d^{2} z \frac{1}{(x-z)^{2}} \\
& \times \operatorname{Tr}\left[T^{a} \widetilde{U}^{\dagger}(x) \widetilde{U}(z)\right] .
\end{aligned}
$$

Here the eikonal scattering amplitudes $U(x)$ and $\widetilde{U}(x)$ are the unitary matrices in the fundamental and adjoint representations, respectively. The noise is characterized by Gaussian local correlations

$$
\begin{gathered}
\text { PACS number(s): 12.38.-t, 25.75.- } \\
\left\langle\xi_{i}^{a}\left(t^{\prime}, z^{\prime}\right) \xi_{j}^{b}\left(t^{\prime \prime}, z^{\prime \prime}\right)\right\rangle=\delta^{a b} \delta_{i j} \delta\left(t^{\prime}-t^{\prime \prime}\right) \delta\left(z^{\prime}-z^{\prime \prime}\right) .
\end{gathered}
$$

This Langevin equation gives rise to an infinite number of equations for correlators of $U$ which coincide with those derived in [2]. In the large $N_{c}$ limit Eq. (1) reduces [3] to a closed equation for the scattering probability $N(x, y)$ of a color singlet dipole with charges at points $x, y[6], N(x, y)$ $=\left(1 / N_{c}\right) \operatorname{Tr}\left\langle 1-U^{\dagger}(x) U(y)\right\rangle$ :

$$
\begin{aligned}
\frac{d}{d t} N(x, y)= & \frac{\alpha_{s} N_{c}}{2 \pi^{2}} \int d^{2} z \frac{(x-y)^{2}}{(x-z)^{2}(y-z)^{2}} \\
& \times[N(x, z)+N(y, z)-N(x, y) \\
& -N(x, z) N(z, y)] .
\end{aligned}
$$

From the first numerical [7-9] and analytical $[10,11]$ studies of the Balitsky-Kovchegov (BK) Eqs. (1), (4) the following consistent picture emerges: Suppose one starts the evolution from the initial condition of small target fields $[N(x, y) \ll 1$ for all $x, y]$. Initially the evolution follows the BFKL equation, since the nonlinear term in Eq. (4) is negligible. As the scattering probability approaches unity, the nonlinear term kicks in and eventually the growth stops as the right-hand side (RHS) of Eq. (4) vanishes for $N(x, y)=1$. The larger dipoles saturate earlier, the smaller dipoles follow at later "time" $t$. These features are contained in the simple parametrization [12]

$$
N(x, y)=1-\exp \left[-(x-y)^{2} Q_{s}^{2}(t)\right] .
$$

The exact dependence of the saturation momentum $Q_{s}(t)$ on rapidity is not known, but both, the numerical results [7] and simple theoretical estimates $[13,11]$ are consistent with the exponential growth of the form $Q_{s}(t)=\Lambda \exp \left[\alpha_{s} c t\right]$, with $c$ of order unity. This physical picture has been anticipated several years ago in [13].

While the BFKL equation leads to an unphysical exponential growth of the scattering probability $N(x, y)$ with $t$, the nonlinearities of Eqs. (1), (4) tame this growth such that $N(x, y)<1$, as required for a probability. The "saturation" of the scattering probability at fixed impact parameter, however, 
does not ensure that the total scattering cross section is unitary in the sense of satisfying the Froissart bound. We hence refer to it as "saturation" rather than "unitarization."

The total inelastic cross section is given by the integral of the scattering probability over the impact parameter. Thus in the saturation regime

$$
\sigma=\pi R^{2}(t)
$$

where $R(t)$ is the size of the region in the transverse plane for which the scattering probability for a given projectile is unity. The Froissart bound requires the radius $R(t)$ to grow at most linearly with $t$. We now present two simple calculations which establish that within the BK evolution the growth of the radius with rapidity is exponential.

First consider the Langevin equation (1). Assume that initially, at rapidity $t_{0}$ the target is black within some radius $R_{0}$. This means that for $|z|<R_{0}$ the matrix $U(z)$ fluctuates very strongly so that it covers the whole group space. We concentrate on a point $x$ which is initially outside of this black region. The matrix $U(x)$ then is close to unity. Thus there is no correlation between $U(x)$ and $U(z)$, and, in the random phase approximation [4] the second term on the right-hand side of Eq. (1) can be set to zero [14]. As the target field ensemble evolves in rapidity, the radius of the black region grows. As long as the point $x$ stays outside the black region we can approximate the Langevin equation by (we drop color indices which are inessential to our argument)

$$
\frac{d}{d t} U(x)=-\sqrt{\frac{\alpha_{s} N_{c}}{\pi^{2}}} \int_{|z|<R} d^{2} z \frac{(x-z)_{i}}{(x-z)^{2}} \xi_{i}(z) .
$$

This equation neglects contributions to $d / d t U$ from gluons originating from outside the black region, and thus slightly underestimates the rate of growth of the radius of the black region. The formal solution of Eq. (6) is

$$
1-U(x, t)=\sqrt{\frac{\alpha_{s} N_{c}}{\pi^{2}}} \int_{t_{0}}^{t} d \tau \int_{|z|<R(\tau)} d^{2} z \frac{(x-z)_{i}}{(x-z)^{2}} \xi_{i}(z) .
$$

Squaring it and averaging over the noise term gives

$$
\left\langle[1-U(x, t)]^{2}\right\rangle=\frac{\alpha_{s} N_{c}}{\pi^{2}} \int_{t_{0}}^{t} d \tau \int_{|z|<R(\tau)} \frac{d^{2} z}{(x-z)^{2}} .
$$

As long as $x$ is outside the black region we can approximate the integral on the right-hand side by

$$
\int_{|z|<R(\tau)} d^{2} z \frac{1}{(x-z)^{2}}=\pi \frac{R^{2}(\tau)}{x^{2}}
$$

and Eq. (8) becomes

$$
\left\langle[1-U(x, t)]^{2}\right\rangle=\frac{\alpha_{s} N_{c}}{\pi} \frac{1}{x^{2}} \int_{t_{0}}^{t} d \tau R^{2}(\tau) .
$$

Now as the black region grows, eventually it reaches the point $x$. At this rapidity the matrix $U(x)$ will start fluctuating with the amplitude of order one. Thus when $R(t)=|x|$, the left-hand side of Eq. (10) becomes a number of order one, which we call $1 / \epsilon$. We thus have an approximate equation for $R(t)$ :

$$
\frac{1}{\epsilon} R^{2}(t)=\frac{\alpha_{s} N_{c}}{\pi} \int_{t_{0}}^{t} d \tau R^{2}(\tau)
$$

At large rapidities therefore the radius of the black region is exponentially large

$$
R(t)=R\left(t_{0}\right) \exp \left[\frac{\alpha_{s} N_{c}}{2 \pi} \epsilon\left(t-t_{0}\right)\right] .
$$

This is our main result.

We note that while the approximations leading to Eq. (11) cease to be valid when the point $x$ is on the boundary of the black region, this does not affect our main conclusion. First, Eq. (9) is an underestimate of the integral, thus underestimating the growth of $R$. Second, when $x$ is on the boundary of the black region and $z$ in the black region, although the factors $\left[1-U(x) U^{\dagger}(z)\right]$ and $U(x)$ in Eq. (1) are not strictly unity, they are still of order one for almost all points $z$. Thus, although we cannot determine the exact numerical value of $\epsilon$, the functional form of the solution as well as its parametric dependence is given correctly by Eq. (12).

Note that Eq. (1) refers to the evolution of the matrix $U(x)$, which can be thought of as the scattering amplitude of a colored probe. However, since the physics of the BK equation does not incorporate effects of confinement, the cross section for a colorless dipole within the BK framework must grow in the same way. To establish this point, and to make more explicit the relation between $\epsilon$ and the BFKL dynamics, we now present an alternative derivation of Eq. (12).

To this end we consider the BK evolution as the evolution of the projectile [3]. Suppose at the initial energy $t_{0}$ the projectile is a color dipole of size $x_{0}$. It scatters on a hadronic target of some size $R_{0}$. As is explicit in [3], as the energy is increased the projectile wave function evolves according to the BFKL equation. Thus at rapidity $t$ the density of dipoles of size $x$ at transverse distance $r$ from the original dipole is given by the BFKL expression (see, for example, [15])

$$
\begin{aligned}
n\left(x_{0}, x, r, t\right)= & \frac{32}{x^{2}} \frac{\ln \frac{16 r^{2}}{x_{0} x}}{\left(\pi a^{2} t\right)^{3 / 2}} \\
& \times \exp \left[\omega t-\ln \frac{16 r^{2}}{x_{0} x}-\frac{\ln ^{2} \frac{16 r^{2}}{x_{0} x}}{a^{2} t}\right]
\end{aligned}
$$

with $\omega=4 \ln 2 N_{c} \alpha_{s} / \pi$ and $a^{2}=14 \zeta(3) N_{c} \alpha_{s} / \pi$. When the density of dipoles at a given impact parameter is greater than one, multiple scatterings become important. Thus the scattering probability is not proportional to $n$, but is an infinite series containing all multiple scattering terms [3].

For our argument, it is only important that once the density of dipoles at some impact parameter $r$ becomes larger than some fixed critical number, the scattering amplitude at 
this impact parameter saturates. The exact value of this number depends on the target, but importantly it does not depend on rapidity. Thus the total cross section is given by the square of the largest impact parameter at which the dipole density in the projectile wave function is of order unity. In order to estimate this directly from Eq. (13), let us choose the dipole size $x$ in Eq. (13) as $x=Q_{s}^{-1}\left(t_{0}\right)$. Recall that according to Eq. (4), the dipole of this size scatters with probability one, if it hits inside the radius of the target $R_{0}$ (in this view of the evolution only the projectile wave function depends on energy, while the properties of the target at $t$ are the same as at $t_{0}$ ). Thus if at some impact parameter $R(t)$ the density of dipoles of size $Q_{s}^{-1}\left(t_{0}\right)$ is unity, the scattering probability at this impact parameter is unity as well. Requiring the exponential in Eq. (13) to vanish we obtain [16]

$$
\begin{aligned}
R^{2}(t) & =\frac{1}{16} \frac{x_{0}}{Q_{s}\left(t_{0}\right)} \exp \left[\frac{\alpha_{s} N_{c}}{\pi} \epsilon t\right], \\
\epsilon & =7 \zeta(3)[-1+\sqrt{1+8 \ln 2 / 7 \zeta(3)}] .
\end{aligned}
$$

Thus, also for a color singlet projectile, we arrive at the exponential growth of the cross section.

The exact value of $\epsilon$ given in Eq. (15) should not be taken too seriously. The explicit form of the dipole density Eq. (13) was derived by a saddle point integration, and as such is valid only for $\ln \left(16 r^{2} / x_{0} x\right)<\alpha_{s} t$. This condition is not satisfied by Eq. (14). However, even beyond the saddle point approximation the density has the form

$$
n\left(x_{0}, x, r, t\right) \propto \frac{1}{x^{2}} \exp \left[\alpha_{s} t F\left(\frac{\ln \frac{16 r^{2}}{x_{0} x}}{\alpha_{s} t}\right)\right] .
$$

The relevant condition is $F=0$. Thus, while our calculation does not specify the numerical value of $\epsilon$, the correct solution parametrically is the same as Eq. (14).

Note, that although we use the BFKL dipole density of Eq. (13), our argument does not assume that the scattering probability at $R(t)$ is dominated by one pomeron exchange. The only assumption is that parametrically the total unitarized probability is the same as the one Pomeron one. In terms of the projectile wave function this translates into the condition $\sum_{m=1}^{\infty} P_{m}\left(x_{0}, x, r, t\right)=c$ whenever $n\left(x_{0}, x, r, t\right)=1$. Here $P_{m}\left(x_{0}, x, r, t\right)$ is the probability to find $m$ dipoles of size $x$ at transverse coordinate $r$ in the projectile wave function and $c$ is a number of order one. The only way this condition can be violated, is if the wave function is dominated (with exponential accuracy) by the trivial configuration with no dipoles, even when the average dipole number is one. Although the dipole model wave function is known to have relatively large fluctuations, there is nothing in its known properties $[6,15]$ to suggest such an extreme behavior. In fact for the explicit exponential model used in $[6,15]$ our condition clearly holds.

Our results, Eqs. (11), (14), are in apparent contradiction with the conclusions of numerical work $[7,8]$. These references solve Eq. (4) within local approximation, assuming that important contributions come only from the dipole sizes which are smaller than the impact parameter. Within this approximation the dependence on the impact parameter in Eq. (4) becomes parametric, and the growth of the total cross section is determined entirely by the shape of the initial condition. The Froissart bound is then saturated for the exponential initial profile of $N(r)$. The reason the local approximation leads to this behavior is that it neglects the effects of far away black regions (where $N=1$ ) on the scattering probability in the gray areas (where $N<1$ ). As is apparent from our analysis in Eqs. (6)-(10), it is precisely the effect of the far away black regions that drives the growth of the total cross section. This is due to the long range Coulomb fields originating in the central black region. In fact, the only contributions we kept on the RHS of Eq. (6) are due to dipoles with sizes of the order of the impact parameter. In this respect our discussion is orthogonal to that of $[7,8]$. The local approximation is adequate for studying the behavior of $Q_{s}(t)$ in the dense central region, as this is determined by local effects. It is, however, not a good approximation for the total cross section, which is dominated by the evolution of long range Coulomb fields.

On the other hand, our results are in agreement with those of [15]. Reference [15] does not deal with the nonlinear BK equations, but rather with the onium-onium scattering in the framework of the dipole model. Nevertheless at asymptotically high energies this distinction should be irrelevant. Indeed the numerical results of [15] clearly indicate that even though the scattering amplitude is unitarized locally in the impact parameter space, the total cross section keeps on growing exponentially with $t$ (Figs. 9 and 10 of [15]).

In the target rest frame, this violation of unitarity by the BK evolution can be understood as follows: Start with a single dipole scattering on the hadronic target of transverse size $R_{0}$. With increasing energy the projectile dipole emits additional dipoles strictly according to the BFKL evolution. The density as well as the transverse size of the projectile state thus grows. The increase in density leads to increasing importance of multiple scatterings which are properly accounted for in the BK derivation. This ensures that the scattering probability saturates locally. In the saturation regime, as long as the size of the projectile state $R(t)$ is smaller than the target size $R_{0}$, the cross section grows essentially only due to surface effects:

$$
\sigma=\pi R_{0}^{2}+2 \pi R_{0} x_{0} \exp \left[\frac{\alpha_{s} N_{c}}{2 \pi} \epsilon t\right] .
$$

Thus as long as $\alpha_{s} \epsilon t<\ln \left(R_{0} / x_{0}\right)$, the cross section is practically geometrical. However, once the energy is high enough so that the projectile size is larger than that of the target, the total cross section is determined by the former and grows exponentially with the logarithm of energy according to Eq. (14).

This also illustrates that the applicability of the BK evolution crucially depends on the nature of the target. If the target is thick enough, so that the multiple scatterings become important before the growth of the projectile radius does, and if the target is wide enough, so that saturation occurs before the projectile radius swells beyond that of the 
target, then there is an intermediate regime in which the inelastic cross section remains practically constant and equal to $\pi R_{0}^{2}$. Then BK applies. However, if the target is a nucleon, neither one of these conditions is satisfied. Thus the tainted infrared behavior of the BFKL evolution of the projectile will show up right away and will invalidate the application of the BK equation.

In order to discuss the violation of unitary from the point of view of the evolution of target fields, we now go back to the stochastic process (1). The RHS of Eq. (1) describes the total Coulomb (Weizsäcker-Williams) field at point $x$ due to the color charge sources at points $z$. Since the noise is stochastic, the color sources are completely uncorrelated both in the transverse plane and in rapidity. For this random source, the square of the total color charge is proportional to the area, and this is precisely the factor $R^{2}$ in Eq. (10). The incoming dipole thus scatters on the Coulomb field created by the large incoherent color charge. Because the Coulomb field is long range, the whole bulk of the region populated by the sources contributes to the evolution and leads to rapid growth of $R$. If the field created by the sources was screened by some mass, the evolution would be unitary. To illustrate this point, we substitute the Coulomb field $(x-z)_{i} /(x-z)^{2}$ in Eq. (1) by an exponentially decaying field $m \exp \{-m \mid x$ $-z \mid\}$. It is straightforward to perform now the same analysis as before. Equation (9) is replaced by

$$
\int_{|z|<R(\tau)} d^{2} z m^{2} \exp \{-m|x-z|\}=\exp \{-m|x-R|\}
$$

This leads to the substitution $R^{2} \rightarrow \exp \{m R\}$ in all subsequent equations with the end result that

$$
R(t)=\alpha_{s} \frac{\epsilon}{m} t
$$

which in fact saturates the Froissart bound. Thus the reason for the violation of unitarity is that the evolution is driven by the emission of the long range Coulomb field from a large number of incoherent color sources in the target.

Cutting off the Coulomb field is not the only possibility to cure this problem. Another option is that the sources of the color charge in the high density regime cease to be incoherent. If they have correlations ensuring that the total color charge in a region of fixed size $L$ is zero, then the incoming dipole would feel the Coulomb field only within the fixed distance $L$ from the black region. Thus the new charges produced by the evolution would only "split off" the edges of the black region rather than from its bulk. This scenario is equivalent to exponential decay of the field, and will lead to a unitary evolution. In a confining theory like QCD, it is likely to be materialized. We note that the desirability of such color charge correlations was stressed in a somewhat different context in [17].

Although such charge correlations do not arise in the BK evolution, it is not a priori clear that they are not present in a more complete semiperturbative framework which still does not take into account the physics of confinement at low energies. The BK framework is incomplete inasmuch as it takes the evolution of the projectile wave function to be pure BFKL. Once the density of gluons in this wave function becomes large, interactions should lead to saturation effects on the wave function level, i.e., the density of the dipoles should grow slower than Eq. (13). Such corrections should still be semiperturbative, in the sense that they are present at small $\alpha_{s}$. For scattering on "small" targets, they become important at the same energy as the multiple scattering terms resummed in Eqs. (1), (4). These wave function saturation effects may lead to charge correlations of the type necessary to unitarize the total cross section.

This work has been supported in part by PPARC. We thank G. Milhano and H. Weigert for helpful discussions.
[1] L.V. Gribov, E. Levin, and M. Ryskin, Phys. Rep. 100, 1 (1983); A. Mueller and J.W. Qiu, Nucl. Phys. B286, 427 (1986)

[2] I. Balitsky, Nucl. Phys. B463, 99 (1996); Phys. Rev. Lett. 81, 2024 (1998); Phys. Rev. D 60, 014020 (1999).

[3] Yu. Kovchegov, Phys. Rev. D 60, 034008 (1999).

[4] H. Weigert, Nucl. Phys. A703, 823 (2002).

[5] Nonlinear evolution equations were also derived in the Wilson renormalization group approach in J. Jalilian Marian et al., Phys. Rev. D 59, 014014 (1999); 59, 014015 (1999); A. Kovner and J.G. Milhano, ibid. 61, 014012 (2000). Whether these results are generally equivalent to Eqs. (1), (4) or incorporate different physics is still an open question. Here, we focus entirely on the BK equations (4), (1).

[6] A. Mueller, Nucl. Phys. B415, 373 (1994); B437, 107 (1995).

[7] E. Levin and K. Tuchin, Nucl. Phys. B573, 833 (2000); Nucl. Phys. A693, 787 (2001).
[8] M.A. Braun, Eur. Phys. J. C 16, 337 (2000).

[9] K. Golec-Biernat, L. Motyka, and A.M. Stasto, Phys. Rev. D 65, 074037 (2002).

[10] Yu. Kovchegov, Phys. Rev. D 61, 074018 (2000).

[11] E. Iancu and L. McLerran, Phys. Lett. B 510, 145 (2001).

[12] K. Golec-Biernat and M. Wüsthoff, Phys. Rev. D 60, 114023 (1999).

[13] A. Mueller, Nucl. Phys. B558, 285 (1999).

[14] Note that this approximation does not linearize the evolution. Rather it corresponds to taking the last (nonlinear) term in Eq. (4) as unity for $z$ in the black region.

[15] G.P. Salam, Nucl. Phys. B461, 512 (1996).

[16] Equation (14) is valid with exponential accuracy. To determine the pre-exponential factor one would have to treat more carefully the pre-exponential factors in Eq. (13). This is beyond the scope of this Rapid Communication.

[17] C.S. Lam and G. Mahlon, Phys. Rev. D 61, 014005 (2000). 